\begin{tabular}{|c|l|}
\hline Title & A nalysis of errors in linear-optics quantum computation \\
\hline Author(s) & Takeuchi, Shigeki \\
\hline Citation & $\begin{array}{l}\text { Physical Review A, 61, 052302 } \\
\text { https://doi.org/10.1103/PhysRevA .61.052302 }\end{array}$ \\
\hline Issue Date & 2000-03-31 \\
\hline Doc URL & http://hdl.handle.net/2115/5560 \\
\hline Rights & Copyright $\odot 2000$ A merican Physical Society \\
\hline Type & article \\
\hline File Information & PRA 61-5.pdf \\
\hline
\end{tabular}

Instructions for use 


\title{
Analysis of errors in linear-optics quantum computation
}

\author{
Shigeki Takeuchi* \\ Mitsubishi Electric Corporation, Advanced Technology R\&D Center, Amagasaki, Hyogo 661-8661 Japan \\ and PRESTO Project, Japan Science and Technology Corporation, Kawaguchi, Saitama, 332-0012 Japan
}

(Received 27 September 1999; published 31 March 2000)

\begin{abstract}
The origin of errors in quantum computation implemented by linear optics is studied. The systematic errors of quantum gates, phase relaxation, amplitude dumping, and misreadout are considered as error sources, and the errors which occurred in a four-bit Deutsch-Jozsa algorithm experiment are categorized according to the sources. The increase in the error rate with the expansion of the input size in the Deutsch-Jozsa algorithm is also studied and it was found that the demonstration of 11 qubits using linear optics and a single photon with less than a $20 \%$ error rate is achievable by the technique used in the experiment.
\end{abstract}

PACS number(s): 03.67.Lx, 42.30.-d

\section{INTRODUCTION}

Quantum computation is a new concept which utilizes quantum superposition states for ultrafast parallel processing $[1-3]$. There have been several proposals for the actual realization of quantum computers [4-6]. Of these, nuclearmagnetic-resonance quantum computation (NMR-QC) has played the role of a test bed for these algorithms $[7,8]$. However, NMR-QC has the following restrictions: the input qubits are prepared in "hot mixed states," and the results are always given by an average over a huge number of quantum systems. Therefore there is a class of algorithms which cannot be performed by NMR-QC [9].

Quantum computation using linear optics and a single photon is an alternative test bed for quantum computation. In contrast to NMR-QC, the initial qubits can be prepared in pure states and the result can be given not by an average but by a single quantum computation. In quantum algorithms, appropriate unitary transformations are applied to quantum registers. Reck et al. found that linear optics can be used to realize any unitary transformation [10]. A theoretical proposal of quantum computation using linear optics was given by Takeuchi [11], and later a similar idea was suggested independently by Cerf et al. [12]. Recently, an experimental demonstration of the four-bit Deutsch-Jozsa (DJ) quantum computation algorithm [13] using linear optics and a single photon whose size was equivalent to 3 qubits was reported [14]. In the experiment, the answer was given with an error rate of less than $8 \%$.

In this paper, we report a quantitative analysis of the sources of error in linear optics computation. In quantum computation, error is caused by several sources: systematic errors in quantum gates, phase relaxation, amplitude dumping, and misreadout. We estimated the effect of each source in the four-bit DJ experiment, and succeeded in reproducing the error rate which appeared in the experiment by using the sum of the estimated error rates according to the sources.

We also studied the increase in the error rate when the size of the input digits is expanded to $2^{k}$ bits and estimated

\footnotetext{
*Present address: RIES, Hokkaido University, Sapporo, 060-0812 Japan.
}

the errors of the expanded system using the error parameters of the four-bit DJ experiment. We concluded that a quantum computation of 11 qubits using linear optics is achievable with less than a $20 \%$ error rate using the technique used in the experiment. When we consider the limit of NMR-QC to be ten qubits, and this result suggests that linear optics quantum computation is one of the most practical test beds for quantum computation.

We also found that the main source of error was caused by the dark counts of the photon detectors when the size of the system was increased, and that the increase in error rates depends on the answers ("not even" and "not uniform") to the DJ problem. We think this kind of error analysis will help to invent a type of algorithm which is tolerant to the source of error.

The rest of the paper is structured into six sections: Section II consists of an introduction to the implementation of quantum computation using linear optics. Section III describes the sources of errors in the linear optics quantum computation and an analysis of the effect of the sources in a simple optical system. Section IV consists of the result of the four-bit DJ experiment together with the experimental setup. In Sec. V, a quantitative analysis of the error in the experiment is given. We discuss the increase in error rate with the number of qubits used in the algorithm in Sec. VI. In Sec. VII, we conclude with a summary of this paper.

\section{OPTICAL IMPLEMENTATION OF QUANTUM COMPUTATION USING LINEAR OPTICS}

Any quantum algorithm is compatible with a proper quantum circuit. Deutsch also showed that any quantum logic gate can be constructed using universal quantum gates, such as rotation gates for a single qubit and controlled NOT gates for two qubits. In the linear optics quantum computation, we use the modes of a single photon for several qubits and polarization for one qubit. For example, if we need $k$ qubits for a quantum computation, we can prepare $k-1$ qubits using $2^{k-1}$ modes and one qubit by polarization. In the following, we show that simple linear optical devices such as beam splitters and phase shifters act as quantum gates for these qubits.

An example of a quantum circuit with a single qubit is 
(a)

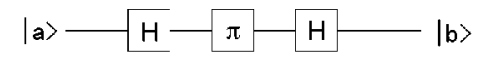

(b)

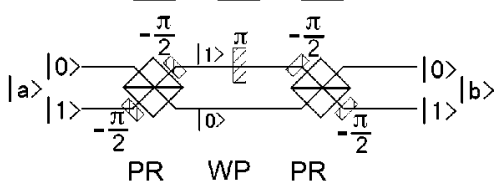

(c)

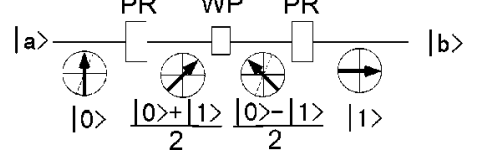

FIG. 1. (a) An example of a quantum circuit using a single qubit, which is equivalent to a NOT gate. (b) An implementation of the quantum circuit using two optical modes. (c) Another implementation using polarization of a single photon.

shown in Fig. 1(a). The quantum circuit contains a $\pi$ phaseshift gate between a pair of Hadamard gates: $|0\rangle$ is transformed to $(|0\rangle+|1\rangle) / \sqrt{2}$ and $|1\rangle$ to $(|0\rangle-|1\rangle) / \sqrt{2}$. When the input qubit $|a\rangle$ is $|0\rangle$, the qubit $|b\rangle$ should be $|1\rangle$. In this sense this circuit works as a NOT gate in total.

An optical implementation of this circuit is the MachZehnder interferometer shown in Fig. 1(b). A qubit is represented by 2 modes, $|0\rangle$ and $|1\rangle$. The transformation of a 50:50 beam splitter is given as follows:

$$
\frac{1}{\sqrt{2}}\left(\begin{array}{ll}
1 & i \\
i & 1
\end{array}\right)
$$

When the beam splitter is supplemented with two $-\pi / 2$ phase shifters in mode $|1\rangle$, it acts on these two modes as in the following transformation [12]:

$$
R=\frac{1}{\sqrt{2}}\left(\begin{array}{cc}
1 & 1 \\
1 & -1
\end{array}\right),
$$

which is same with the Hadamard transformation. Suppose the initial qubit $|a\rangle=|0\rangle$ by putting a single photon into input mode $|0\rangle$, we will detect the photon in the output mode $|1\rangle$. Here the path difference between the two modes should be adjusted to 0 .

Figure 1(c) is another example of implementation. In this case, the polarization of a photon is used to implement a qubit: horizontal polarization corresponds to $|0\rangle$ and vertical polarization to $|1\rangle$. Each of the two Hadamard gates is realized by a $\pi / 2$ phase shifter whose optical axis (shown as a dotted line) was set at $22.5^{\circ}$ from the vertical axis, and a $\pi / 2$ phase shifter was implemented by another $\lambda / 2$ wave plate which acts as an optical delay between the vertical and horizontal polarizations.

Another example of a quantum circuit of two qubits shown in Fig. 2(a). When the state $|a\rangle|b\rangle=|0\rangle|0\rangle$ is input, the output state will be a uniform superposition of the states; $|a\rangle|b\rangle=(|0\rangle|0\rangle+|0\rangle|1\rangle+|1\rangle|0\rangle+|1\rangle|1\rangle) / 2$. The optical system uses four modes to implement these two qubits. The first Hadamard transformation is realized by beam splitter 1 (BS1) and BS2, and the second one is realized by BS3 and BS4. This implementation is used as a part of the optical (a)

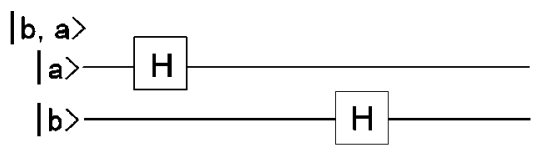

(b)

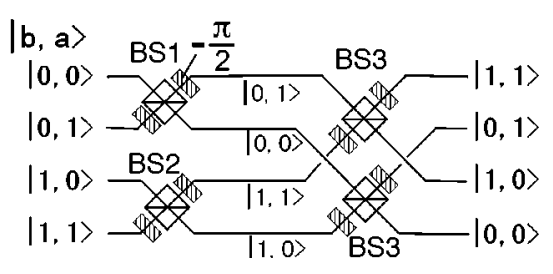

FIG. 2. (a) An example of a quantum circuit of two qubits. (b) An implementation of the quantum circuit using four optical modes.

system in the four-bit DJ experiment. Another implementation is also possible using polarization for qubit $|A\rangle$, and two modes for qubit $|B\rangle$.

Linear optics quantum computation has some advantages over NMR-QC's: we can prepare initial qubits in a pure state or any mixed state, and we can also perform "'a single quantum computation", using a single photon. It was also shown that any quantum algorithm can be implemented by linear optics [12]. Although it may not be suitable for large-scale computation because it requires $2^{N-1}$ paths for the demonstration with $N$ qubits, it will be used as an alternative test bed of quantum computing.

\section{SOURCES OF ERROR IN LINEAR OPTICS QUANTUM COMPUTATION}

Here we study the sources of error in the linear optics quantum computation. The main sources of error in quantum computation can be listed as follows: (1) Systematic error of quantum gates, (2) phase relaxation, (3) amplitude dumping, and (4) misreadout of the computation result.

In quantum circuits, qubits are rotated by a given angle at quantum gates. "The systematic error of quantum gates" is the difference between the actual rotation angle and the supposed one. "Phase relaxation" means the broadening of the phase distribution of the wave function. The phase relaxation squashes the quantum interference, and increases the error of the computation. In linear optics quantum computation, the surface roughness of the optics, the fluctuation of the reflective index of the air, and the misalignment of the optics are of this type. "Amplitude dumping" of the wave function occurs when photons are absorbed or reflected unintentionally in the optical system. "Misreadout" corresponds to the dark counts in the experiment using a single photon. Some of them are caused by stray photons and some by the intrinsic noise of the photon detector.

In this section, we analyze the effect of these sources in the simple optical system shown in Fig. 3. This optical system is a Michelson interferometer which is equivalent to the system shown in Fig. 1(b). A single photon is put into mode 1 of a 50:50 beam splitter and the result is detected by a photon detector at output mode 1 or 2 . When the path length $x_{1}$ and $x_{2}$ are set to be equal and no error source exists, the photon should be detected by detector D1 at output mode 1 . In the following, we consider nonideal cases with error sources. 


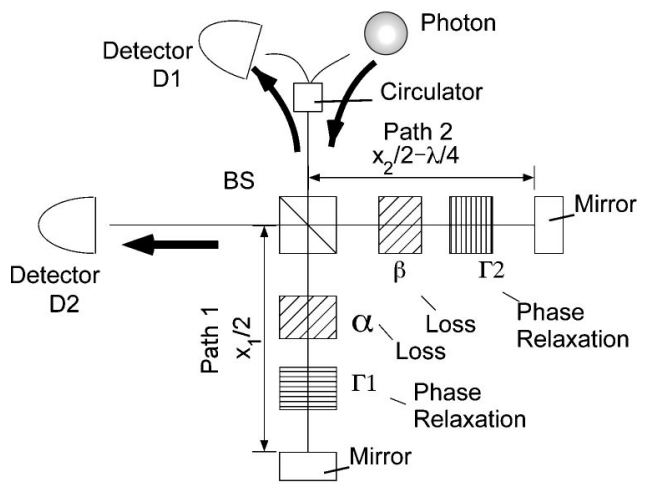

FIG. 3. A schematic diagram of the optical system for the quantum circuit shown in Fig. 1(a).

\section{A. The systematic errors of quantum gates}

In the optical system shown in Fig. 3, the two Hadamard gates in Fig. 1(a) are implemented by a beam splitter BS. In this case, the error of the splitting ratio of the beam splitter is one of the systematic errors. The transformation of the beam splitter with a pair of $-\pi / 2$ phase shifters in mode $|1\rangle$ is given as follows:

$$
R(\theta)=\left(\begin{array}{cc}
\cos \frac{\theta}{2} & \sin \frac{\theta}{2} \\
\sin \frac{\theta}{2} & -\cos \frac{\theta}{2}
\end{array}\right),
$$

where $\theta$ corresponds to the rotation angle of a qubit.

There is another source of systematic error. When the path difference is not adjusted to be 0 (or the supposed value), it is equivalent to where an unnecessary phase-shift gate is added to the optical system. The effect of the path difference is given by the following transformation $L\left(x_{1}, x_{2}\right)$ :

$$
L\left(x_{1}, x_{2}\right)=\left(\begin{array}{cc}
\exp \left(\frac{2 \pi i x_{1}}{\lambda}\right) & 0 \\
0 & \exp \left(\frac{2 \pi i x_{2}}{\lambda}\right)
\end{array}\right),
$$

where $x_{1}, \lambda, x_{2}$ mean twice the optical length of path 1 , the wave length of the incident photon, and twice the optical length of path 2 plus $\lambda / 2$. Using these two equations, the wave function at the output mode is given as follows:

$$
\begin{aligned}
\left(\begin{array}{l}
\psi_{1} \\
\psi_{2}
\end{array}\right) & =R(\theta) L\left(x_{1}, x_{2}\right) R(\theta)\left(\begin{array}{l}
1 \\
0
\end{array}\right) \\
& =\left(\begin{array}{l}
\exp \left(i k x_{1}\right) \cos ^{2} \frac{\theta}{2}+\exp \left(i k x_{2}\right) \sin ^{2} \frac{\theta}{2} \\
{\left[\exp \left(i k x_{1}\right)-\exp \left(i k x_{2}\right)\right] \sin \frac{\theta}{2} \cos \frac{\theta}{2}}
\end{array}\right),
\end{aligned}
$$

where $k=2 \pi / \lambda$. When $\theta=\pi / 2$ and $k\left(x_{1}-x_{2}\right)=\pi$, the output state ${ }^{t}\left(\psi_{1}, \psi_{2}\right)$ becomes ${ }^{t}(0,1)$ which is equivalent to the result of the quantum circuit shown in Fig. 1 without any error. The detection probability at the output modes is given as follows:

$$
\begin{aligned}
P_{1} & =\psi_{1}^{*} \psi_{1} \\
& =1-\frac{1}{2}\left[1-\cos k\left(x_{1}-x_{2}\right)\right] \sin ^{2} \theta, \\
& P_{2}=\frac{1}{2}\left[1-\cos k\left(x_{1}-x_{2}\right)\right] \sin ^{2} \theta .
\end{aligned}
$$

First let us consider the case where the parameters should be set as $\theta=\pi / 2$ and $x_{1}-x_{2}=0$. The probabilities are presumed to be $P_{1}=1, P_{2}=0$. When we have systematic errors, these parameters are given as $\theta=\pi / 2+\delta_{\theta}$ and $k\left(x_{1}-x_{2}\right)$ $=\delta_{\beta}$. In this case, the error rate $E(0)$ is given as follows:

$$
\begin{aligned}
E(0) & =\left(1-P_{1}\right)=P_{2} \\
& =\frac{1}{2}\left(1-\cos \delta_{\beta}\right) \cos ^{2} \delta_{\theta} \\
& \sim \frac{1}{4} \delta_{\beta}^{2} .
\end{aligned}
$$

In the last approximation, we neglected the terms higher than the second order of error. Similarly, when we consider the case where the parameters should be set as $\theta=\pi / 2$ and $k\left(x_{1}-x_{2}\right)=\pi$, the error rate $E(\pi)$ is written by

$$
\begin{aligned}
E(\pi) & =P_{1}=\left(1-P_{2}\right) \\
& =1-\frac{1}{2}\left(1+\cos \delta_{\beta}\right) \cos ^{2} \delta_{\theta} \\
& \sim \frac{1}{4} \delta_{\beta}^{2}+\delta_{\theta}^{2} .
\end{aligned}
$$

\section{B. The effect of phase relaxation}

Next, let us study the phase relaxation of the wave function, which results in the increase of error through the loss of the quantum interference effect. In an extreme case when the distribution of the phase is uniform from 0 to $2 \pi$, no interference appears. In linear optics quantum computation, the main causes of the phase relaxation are (a) the surface roughness of the optics, (b) the fluctuation in the reflective index of air, and (c) an imperfect overlap of the wave front because of the misalignment of the optics. These processes can be taken into account using a phase parameter $\delta_{j}$ in the transformation $L\left(x_{1}, x_{2}\right)$ :

$$
L^{\prime}\left(x_{1}, x_{2}\right)=\left(\begin{array}{cc}
\exp \left(\frac{2 \pi i x_{1}}{\lambda}+\delta_{1}\right) & 0 \\
0 & \exp \left(\frac{2 \pi i x_{2}}{\lambda}+\delta_{2}\right)
\end{array}\right)
$$


Because (a) and (b) are stochastic processes, we may assume $\delta_{j}$ has a Gaussian distribution. For (c) we may also assume a Gaussian distribution when the photon number density has a Gaussian distribution in its radius. Therefore, we assume $\delta_{j}$ is a Gaussian distribution $\omega\left(\delta_{j}, \Gamma_{j}\right)$,

$$
\omega\left(\delta_{j}, \Gamma_{j}\right)=\left(\frac{1}{4 \pi \Gamma_{j}}\right)^{1 / 2} e^{-\left(\delta_{j}^{2} / 4 \Gamma_{j}\right)} .
$$

Calculating the output state ${ }^{t}\left(\psi_{1}, \psi_{2}\right)$ using Eq. (5) with $L^{\prime}\left(x_{1}, x_{2}\right)$ instead of $L\left(x_{1}, x_{2}\right)$, the photon detection probability at output mode 1 is given as follows:

$$
\begin{aligned}
P_{1} & =\int_{-\infty}^{\infty} \omega\left(\delta_{1}, \Gamma_{1}\right) \int_{-\infty}^{\infty} \omega\left(\delta_{2}, \Gamma_{2}\right) \psi_{1}^{*} \psi_{1} \mathrm{~d} \delta_{1} \mathrm{~d} \delta_{2} \\
& =1-\frac{1}{2}\left[1-\exp \left(-\Gamma_{1}-\Gamma_{2}\right) \cos (k \delta x)\right] \sin ^{2} \theta
\end{aligned}
$$

As shown above, the total phase relaxation parameter of the system $\Gamma$ is given by $\Gamma_{\text {total }}=\Gamma_{1}+\Gamma_{2}$. Similarly, when we have an additional source of phase relaxation, we can have $\Gamma_{\text {total }}$ by just summing them.
In this case, the error rate $E(0)$ and $E(\pi)$ are calculated as follows:

$$
\begin{gathered}
E(0)=\frac{1}{4} \delta_{\beta}^{2}+\frac{1}{2} \Gamma_{\text {total }}, \\
E(\pi)=\delta_{\theta}^{2}+\frac{1}{4} \delta_{\beta}^{2}+\frac{1}{2} \Gamma_{\text {total }} .
\end{gathered}
$$

\section{Amplitude dumping}

The amplitude dumping of wave functions corresponds to the optical loss in linear optics quantum computation. We adopt the following matrix which describes the unbalanced loss in each path of the interferometer:

$$
N_{D}=\left(\begin{array}{cc}
\exp (-\alpha) & 0 \\
0 & \exp (-\beta)
\end{array}\right) .
$$

Then the wave function at the output mode of the interferometers with loss is given as follows:

$$
\begin{aligned}
\left(\begin{array}{l}
\psi_{1} \\
\psi_{2}
\end{array}\right) & =R(\theta) N_{D} L\left(x_{1}, x_{2}\right) R(\theta)\left(\begin{array}{l}
1 \\
0
\end{array}\right) \\
& =\left(\begin{array}{c}
\exp (-\alpha) \exp \left(i k x_{1}\right) \cos ^{2} \frac{\theta}{2}+\exp (-\beta) \exp \left(i k x_{2}\right) \sin ^{2} \frac{\theta}{2} \\
{\left[\exp (-\alpha) \exp \left(i k x_{1}\right)-\exp (-\beta) \exp \left(i k x_{2}\right)\right] \sin \frac{\theta}{2} \cos \frac{\theta}{2}}
\end{array}\right) .
\end{aligned}
$$

The detection probability $P_{1}$ and $P_{2}$ is given by,

$$
\begin{gathered}
P_{1}=e^{-2 \alpha} \cos ^{4} \frac{\theta}{2}+e^{-2 \beta} \sin ^{4} \frac{\theta}{2}+\frac{1}{2} e^{-\alpha-\beta} \sin ^{2} \theta \cos (k \delta x), \\
P_{2}=\frac{1}{2} \sin ^{2} \theta\left(\frac{e^{-2 \alpha}+e^{-2 \beta}}{2}-e^{-\alpha-\beta} \cos (k \delta x)\right)
\end{gathered}
$$

On the other hand, the output from an interferometer with a balanced loss $\exp (-A)$ and a phase relaxation $\Gamma$ can be written as follows:

$$
\begin{gathered}
P_{1}=\exp (-A)\left(1-\frac{1}{2}[1-\exp (-\Gamma) \cos k \delta x] \sin ^{2} \theta^{\prime}\right) \\
P_{2}=\exp (-A)\left(\frac{1}{2}[1-\exp (-\Gamma) \cos k \delta x] \sin ^{2} \theta^{\prime}\right) .
\end{gathered}
$$

When we compare these equations for the terms of $\cos (k \delta x)$, we have the following relations:

$$
\begin{gathered}
\exp (-A)=\exp (-2 \alpha) \cos ^{2} \frac{\theta}{2}+\exp (-2 \beta) \sin ^{2} \frac{\theta}{2}, \\
\exp \left(-\Gamma^{\prime}\right)=\frac{2 e^{-\alpha-\beta}}{e^{-2 \alpha}+e^{-2 \beta}}, \\
\sin ^{2} \theta^{\prime}=\sin ^{2} \theta \frac{e^{-2 \alpha}+e^{-2 \beta}}{2\left(e^{-2 \alpha} \cos ^{2} \frac{\theta}{2}+e^{-2 \beta} \sin ^{2} \frac{\theta}{2}\right)} .
\end{gathered}
$$

It is interesting to see that the output of the Michelson interferometer with unbalanced loss is the same as that whose loss is balanced but where phase relaxation and beam splitting ratio errors occur. However, note that the output state ${ }^{t}\left(\psi_{1}, \psi_{2}\right)$ is different. The output state form the interferometer with an unbalanced loss still maintains coherence. Therefore, this interpretation of the unbalanced loss is not adaptable in general. The error rate with loss is discussed in the next subsection. 


\section{Weak light as the source of photons}

As an easy way to put single photons into the optical system, we may use weak coherent light. In this case, photons are input to the system randomly. We cannot know when each of them is injected and a single quantum computation is performed by the photon. One way to solve this problem is to set photon detectors on all the output modes of the beam splitters. In this example, we set two detectors D1 and D2. A quantum computation was performed when one of them detected a photon.

First, let us calculate the error rate assuming that the detectors have $100 \%$ quantum efficiency and no dark counts. When we assume $N_{\text {in }}$ photons are put into the system, the numbers of counts by detectors D1 and D2 are given by $N_{\text {in }} P_{1}$ and $N_{\text {in }} P_{2}$, therefore $N_{\text {total }}$ is given by $N_{\text {total }}$ $=N_{i n}\left(P_{1}+P_{2}\right)$. When we consider $E(0)=N_{i n} P_{2} / N_{\text {total }}$ and $E(\pi)=N_{\text {in }} P_{1} / N_{\text {total }}$, the total loss $\exp (-A)$ appears in the numerator and the denominator commonly and is diminished. Therefore, in this simple case, the error rates $E(0)$ and $E(\pi)$ are given by Eqs. (19) and (20) with Eqs. (28)-(30). Note that when the loss is balanced, i.e., when $\alpha=\beta$, the amplitude dumping cause no errors.

Next, let us consider the effect of the quantum efficiency and the dark count of the detectors. When $N_{\text {in }}$ photons are put into input mode 1 , the photon counting rates $N_{1}$ and $N_{2}$ at each detector are as follows:

$$
\begin{aligned}
& N_{1}=N_{0} \eta_{1} P_{1}+N_{1}^{\text {dark }}, \\
& N_{2}=N_{0} \eta_{2} P_{2}+N_{2}^{\text {dark }},
\end{aligned}
$$

where $N_{1}^{\text {dark }}, N_{2}^{\text {dark }}$ are the dark count rates and $\eta_{1}$ and $\eta_{2}$ are the quantum efficiencies of the detectors. The total count rate $N_{\text {total }}$ is given by $N_{\text {total }}=N_{1}+N_{2}$. For simplicity, let us assume that systematic error, phase relaxation, and loss do not exist. In this simple case, $E(0)$ and $E(\pi)$ can be given as follows:

$$
\begin{aligned}
& E(0)=\frac{N_{2}^{\text {dark }}}{N_{0} \eta_{1}+N_{1}^{\text {dark }}+N_{2}^{\text {dark }}}, \\
& E(\pi)=\frac{N_{1}^{\text {dark }}}{N_{0} \eta_{1}+N_{1}^{\text {dark }}+N_{2}^{\text {dark }}} .
\end{aligned}
$$

\section{E. Another error analysis method based on the superposition of modes with error parameters}

In the above subsections, we used matrices for each optics, path differences, and losses. However, when the size of the optical system increases, the number of matrices and parameters which represents errors become huge, and it becomes difficult to categorize the error according to the type of source. In this section, we introduce another method which is convenient for analyzing errors in an optical system with many optics.

In Eq. (23), $\psi_{1}$ is written in the superposition of wave functions of each path $\exp (-\alpha) \cos ^{2}(\theta / 2) \exp \left(i k x_{1}\right)$ and $\exp$ $(-\beta) \sin ^{2}(\theta / 2) \exp \left(i k x_{2}\right)$. We may write these wave functions as

$$
\phi_{j}=a_{j} \exp \left[i\left(\alpha_{j}+\delta_{j}\right)\right]
$$

then we can calculate the photon detection probability $P_{1}$ as follows:

$$
\begin{aligned}
P_{1} & =\int_{-\infty}^{\infty} \int_{-\infty}^{\infty} \prod_{i=1}^{2} \omega\left(\delta_{j}, \Gamma_{j}\right)\left|\sum_{j=1}^{2} \phi_{j, f(j)}\right|^{2} \mathrm{~d} \delta_{1} \mathrm{~d} \delta_{2} \\
& =\sum_{i=1}^{2} a_{i}^{2}+2 a_{1} a_{2} \exp \left(-\Gamma_{1}-\Gamma_{2}\right) \cos \left(\alpha_{1}-\alpha_{2}\right) .
\end{aligned}
$$

Let us consider the error of an interferometer whose path difference is set at 0 . In this case, the error rate $E(0)$ is given as follows:

$$
\begin{gathered}
E(0)=1-P_{1} \\
=E_{1}(0)+E_{2}(0), \\
E_{1}(0)=1-\sum_{i=1}^{2} a_{i}^{2}+2 a_{1} a_{2}-\cos \left(\alpha_{1}-\alpha_{2}\right), \\
E_{2}(0)=2\left[1-\exp \left(-\Gamma_{1}-\Gamma_{2}\right)\right] a_{1} a_{2} \cos \left(\alpha_{1}-\alpha_{2}\right),
\end{gathered}
$$

where $E_{1}(0)$ represents the effect of the systematic errors, and $E_{2}(0)$ shows the effect of phase relaxation. When we consider the case $\theta=\pi / 2+\delta_{\theta}$ and $k\left(x_{1}-x_{2}\right)=\delta_{\beta}$, then we have $a_{1}=\left(1-\sin \delta_{\theta}\right) / 2$ and $a_{2}=\left(1+\sin \delta_{\theta}\right) / 2$. Then, we have $E_{1}(0)=\delta_{\beta}^{2} / 4$ and $E_{2}(0)=\Gamma / 2$, respectively, which is consistent with the result given in Eq. (19).

This method is especially useful to categorize the errors for the optical system in which modes are symmetrical.

\section{FOUR-BIT DJ EXPERIMENT}

In this section, we describe the experiment which demonstrated the DJ algorithm with four-bit input reported elsewhere [14], which is modified to explain the details sufficiently to analyze the source of errors in the experiment.

\section{A. Deutsch-Jozsa problem}

First, let us introduce the problem of the Deutsch-Jozsa algorithm. Suppose we are given an array of $2 N$ digits. We call the arrays "even" when they include as many 1's as 0's (example $\{1,0,1,0\}$ for $N=2$ ), and “uniform'" when they are filled with only 0 's or 1 's (example $\{1,1,1,1\}$ ). The problem for the Deutsch-Jozsa algorithm is to find the correct answer between "the given array is not even"' and "the given array is not uniform." When the array satisfies both cases, either of them can be the answer. A classical computer needs $N$ +1 steps in the worst case, however, a quantum computer can find the answer with $O(\ln (N))$ steps [13]. ${ }^{1}$

\footnotetext{
${ }^{1} O$ is Landau's symbol: $h(x)=O(g(x))$ means $h(x) / g(x)$ is bounded for $x \rightarrow \infty$.
} 


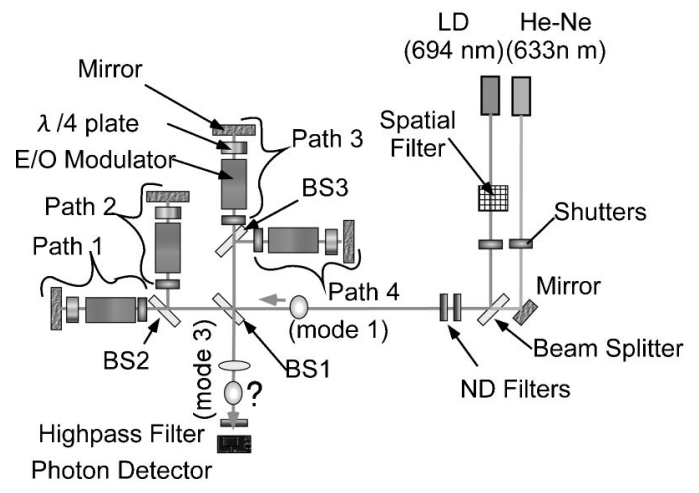

FIG. 4. A schematic diagram of the optical system for the Deutsch-Jozsa algorithm with four-bit inputs.

Our quantum computer solved the problem with four-bit inputs, for which three qubits are required in the DeutschJozsa algorithm; two qubits were used as the address register and 1 as the accumulator for the given oracle. In the computer, four optical paths were used for the address register and the polarization of the photon was used for the accumulator.

The computation was performed as follows. First, the computer is initialized for the computation. Second, "the oracle" (a four-bit digit) was given to the computer and is converted to the appropriate voltage applied to the electrooptic (E/O) modulators in the system. Then, we put a singlephoton with vertical polarization to the input port of this "quantum computer," and observed the detector at the output port to find whether it detected the photon or not. If the photon is detected, the answer is that the given oracle $\{f(i)\}$ is not even. If the photon is not detected, the answer is that the given oracle $\{f(i)\}$ is not uniform. The reason why this computer works will be explained in the next section.

\section{B. Experimental setup}

The experimental setup is shown in Fig. 4. A vertically polarized $694 \mathrm{~nm}$ beam from a laser diode passes through the spatial filter and was attenuated by the neutral density filters to very weak light (up to $0.5 \mathrm{pW}$ ). When such a weak beam was used, the average number of photons present in the optical system (length of each path is $1 \mathrm{~m}$ ) was $3 \times 10^{-3}$. And the probability to find two or more photons in the coherent length of $10 \mathrm{~cm}$ ( width of a single photon wave packet) was less than $3 \times 10^{-4}$. In this sense, the computation was performed using the quantum phenomenon of single-photon interference. All shutters in the optical system except the one for the reference light were open during the computation.

After passing through three beam splitters, the wave function of a single photon is converted to a uniform superposition of the states passing through four optical paths. When the beam splitters are 50/50, the transformation of these three beam splitters is given using Eq. 1 as follows:

$$
\frac{1}{2}\left(\begin{array}{cccc}
1 & i \sqrt{2} & i & 0 \\
i & \sqrt{2} & -1 & 0 \\
i & 0 & 1 & i \sqrt{2} \\
-1 & 0 & i & \sqrt{2}
\end{array}\right)
$$

Therefore, the wave function of the photon after these beam splitters can be described as follows [11]:

$$
I_{1}=\left[\begin{array}{l}
a_{1} \\
a_{2} \\
a_{3} \\
a_{4}
\end{array}\right][p]=\frac{1}{2}\left[\begin{array}{r}
1 \\
i \\
i \\
-1
\end{array}\right][0]
$$

where $a_{i}$ describes the amplitude of the wave function at Path $i$ shown in Fig. 4, and $[p]$ describes the state of the polarization; [0] is the basis of the vertical polarization, and [1] is that of the horizontal polarization. The phase factors according to the optical length of each path will be considered later. This transformation is equivalent to the implementation of the quantum circuit shown in Fig. 2(b) except for the phase factor. We use $\mathrm{E} / \mathrm{O}$ modulators (Gsenger PM0202s) to embed the oracle $\{f(j)\}$ in the system. The modulators rotate the polarization of photons when and only when $f(j)=1$. After the modulation, the wave function becomes:

$$
\frac{1}{2}\left(\left[\begin{array}{l}
1 \\
0 \\
0 \\
0
\end{array}\right][f(1)]+i\left[\begin{array}{l}
0 \\
1 \\
0 \\
0
\end{array}\right][f(2)]+i\left[\begin{array}{l}
0 \\
0 \\
1 \\
0
\end{array}\right][f(3)]-\left[\begin{array}{l}
0 \\
0 \\
0 \\
1
\end{array}\right][f(4)]\right)
$$

Next, quarter-wave plates act as phase shifters, which change the phase of the wave function by $\pi / 2$ only when the polarization of the photons is vertical. After the phase shifters, the mirrors reflect back the wave function. The wave plates added another $\pi / 2$ phase shift according to the state of polarization. Then the $\mathrm{E} / \mathrm{O}$ modulators rotate the polarization again to the original state. The state of the photon after the $\mathrm{E} / \mathrm{O}$ modulators is written as follows:

$$
I_{2}=\frac{1}{2}\left[\begin{array}{r}
(-1)^{f(1)} \\
i(-1)^{f(2)} \\
i(-1)^{f(3)} \\
-(-1)^{f(4)}
\end{array}\right][0]
$$

The transformation of three beam splitters can be written as follows:

$$
\frac{1}{2}\left(\begin{array}{cccc}
1 & i & i & -1 \\
i \sqrt{2} & \sqrt{2} & 0 & 0 \\
i & -1 & 1 & i \\
0 & 0 & i \sqrt{2} & \sqrt{2}
\end{array}\right)
$$

After passing the three beam splitters again, the component $\psi_{3,[0]}$ of the wave function at output mode 3 with vertical polarization $([0])$ is written as 


$$
\begin{aligned}
\psi_{3,[0]} & =i(-1)^{f(1)}-i(-1)^{f(2)}+i(-1)^{f(3)}-i(-1)^{f(4)} \\
& =\sum_{j=1}^{4}(-1)^{f(j)} \exp \left(i \phi_{j}\right)
\end{aligned}
$$

where $\phi_{j}$ is the phase corresponding to the optical path length of path $j$ and the additional phase factors due to the reflection at the beam splitters.

Before the computation we adjusted the path length according to the following initialization procedure. Switching the shutters in the paths, and setting $f(1)=f(4)=1$ and $f(2)=f(3)=0$, the visibilities of the interference between Path 2 and Path 4 , Path 1 and Path 2, and Path 3 and Path 4 are observed sequentially and the tilt angles of the mirrors were adjusted to obtain the maximum visibilities, which were up to $98 \%$. Next, we control the length of Path 4 to set the interferometers to a dark condition (almost no photons observed at the output port) with $0.5 \mathrm{~nm}$ precision using piezoactuators attached directly to each of the mirrors. Using Eq. (48), the output of the interference between Path 2 and Path 4 is proportional to

$$
\begin{aligned}
& \left|(-1)^{f(2)} \exp \left(i \phi_{2}\right)+(-1)^{f(4)} \exp \left(i \phi_{4}\right)\right|^{2} \\
& =2\left[1-\cos \left(\phi_{2}-\phi_{4}\right)\right] .
\end{aligned}
$$

Therefore, setting the interferometer to the dark condition corresponds to having the condition $\phi_{2}=\phi_{4}$. In the same way, we realized the condition $\phi_{1}=\phi_{2}$ and $\phi_{3}=\phi_{4}$ by setting the interference between Path 1 and Path 2, and Path 3 and Path 4 to the dark condition, respectively. The interference of the reference light $(632.8 \mathrm{~nm})$ was used for the precise control of the path lengths. The optical system was also passively stabilized against thermal drifts for at least $10 \mathrm{~s}$ without active control.

Because the phase factors $\phi_{j}$ in Eq. (48) are set to be identical in the initialization procedure, the probability of the detection of the photon at the output port is written as

$$
P(\{f(j)\})=\frac{1}{16}\left|\sum_{j=1}^{4}(-1)^{f(j)}\right|^{2},
$$

where $P=0$ for the "even" input, and 1 for the "uniform", input.

We used a single-photon counting module (SPCM-AQ, EG\&G) as the photon detection device. The observed dark count in the experimental set up was $2 \times 10^{3}$ counts/s on average.

For a given oracle, the photons were counted for $0.1 \mathrm{~s}$. By running the initialization procedure at intervals of $10 \mathrm{~s}$, we succeeded in continuing computation for tens of minutes. This initialization procedure was automatically performed by a personal computer, which also generated the four-bit digits and collected the data.

When the answer of our quantum computer for the given input is wrong, it is termed an "error.' The error rate can be calculated by the photon detection probability $P(\{f(j)\})$ with the given oracle $\{f(j)\}$.

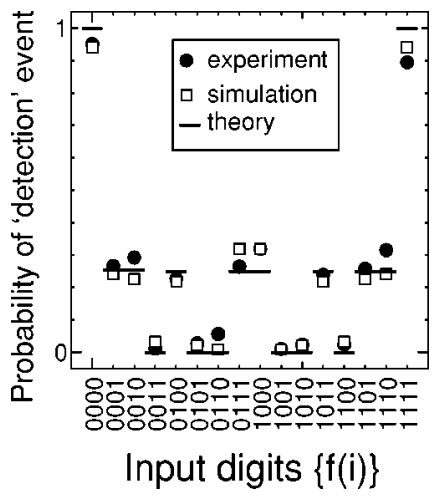

FIG. 5. The photon detection probability for the given four-bit digits $\{\mathrm{f}(\mathrm{i})\}$. The theoretical values with no errors are shown by solid lines, the experimental values are plotted as black dots, and the result of the simulation is plotted as empty squares.

When we use weak light as the source of photons, we somehow have to know when a photon is not detected. One solution is to put three other photon detectors at the open output of the beam splitters. When we observe the photon detection signal from them, we regard it as an event "nondetection.' In this experiment we set the detector only at the output port. In order to derive the detection probability,

$$
P(\{f(j)\})=\frac{N(\{f(j)\})}{N_{\text {total }}},
$$

where $N(\{f(j)\})$ is the photon counting rate with the oracle $\{f(j)\}$, we have to estimate $N_{\text {total }}$ which is the number of total "photon detection events" of all detectors. As is explained in a later section, we estimated $N_{\text {total }}$ as $6.00 \times 10^{5}$ counts/s by $\left[3 N_{d e t}(\right.$ even $)+N_{\text {det }}($ uniform $\left.)\right]$, where $N_{\text {det }}$ (even) and $N_{\text {det }}$ (uniform) were averages of the count rates for the even inputs and uniform inputs. The experimental results are shown in Fig. 5. The vertical axis shows the four-bit digits given to the computer. The horizontal axis shows the probability $P$ of photon observation at the output port. The theoretical values in an ideal condition without any error sources given by Eq. (50) are shown by the solid lines in Fig. 5. The experimental values plotted as the black dots were calculated by Eq. (51).

This result shows that we can determine whether the statement "the given oracle $\{f(j)\}$ is not even" or "the given oracle $\{f(j)\}$ is not uniform"' is correct with the average error rates of $2.57 \%$ and $7.72 \%$, respectively, by the observation of a single photon.

\section{QUANTITATIVE ANALYSIS OF THE ERROR IN THE FOUR-BIT DJ EXPERIMENT}

In this section, we analyze the errors in the four-bit DJ experiment quantitatively according to the sources which are considered in Secs. III. In Secs. III A to III D, we analyzed the optical system corresponding to the quantum circuit of a single qubit using $2 \times 2$ matrices onto 2 optical modes. Similarly, it is possible to analyze the system of the four-bit DJ experiment using $8 \times 8$ matrices. However, when we con- 
sider the transformations by optics one by one, the number of parameters each of which represents an error caused by each of the optics is huge and it is not convenient to classify the errors according to the type of source. Therefore in the following analysis, we analyze the system by the path representation method we introduced in Sec. III E.

In the experiment, we used weak coherent light as the source of photons and we just detected the photon at the output mode. In order to derive the errors of a single quantum computation, we assume in the following analysis that the additional detectors had been set to the other three output modes, and their quantum efficiency and dark count rates were the same as the detector set at the output mode. We call an event "detection" when a photon is detected by D1, and a "nondetection" when detected by the other detectors.

We also assume that the losses in each arm are balanced. Under these assumptions, as we have already seen in Sec. III, the effect of loss in the optical system and the quantum efficiency can be considered simply as the reduction of the number of input photons. In the following, we first estimate the photon counting rate at the detector in the output mode, and derive the average error rate for the case when the input digits are even or uniform. Then we categorize the errors which occurred in the experiment quantitatively.

\section{A. Detection probability of the signal photon}

In Sec. III E, we used two states, $\phi_{1}$ and $\phi_{2}$, to analyze the Mach-Zehnder interferometer. Similarly we use four states $\phi_{j, f(j)}$ according to the path $j$ in Fig. 4, where $f(j)$ is the $j$ th digit of the input. Now $\phi_{j, f(j)}$ is given as follows:

$$
\begin{aligned}
& \phi_{j, f(j)}=a_{j, f(j)} e^{i\left(\alpha_{j, f(j)}+\delta_{j}\right)}, \\
& \alpha_{j, f(j)}=\beta_{j}+f(j)\left(\pi+\gamma_{j}\right),
\end{aligned}
$$

where $a_{j, f(j)}, \alpha_{j, f(j)}$ are the amplitude and phase of the wave function. Errors in the phase $\alpha_{j, f(j)}$ can be described with $\beta_{j}$ which is independent of $f(j)$ and $\gamma_{j}$ which should be added only when $f(j)=1 . a_{j, f(j)}$ does not include the effect of amplitude dumping by balanced loss.

The detection probability of the signal photon can be calculated as $\eta \exp (-A) P_{1}$, where $\eta$ is the quantum efficiency of the detectors, $A$ is a real parameter which corresponds to the effect of balanced loss, and $P_{1}$ is given as follows:

$$
\begin{aligned}
P_{1} & =\int_{-\infty}^{\infty} \ldots \int_{-\infty}^{\infty} \prod_{i=1}^{4} \omega\left(\delta_{i}, \Gamma_{i}\right)\left|\sum_{i} \phi_{i, f(i)}\right|^{2} \mathrm{~d} \delta_{1} \ldots \mathrm{d} \delta_{4} \\
& =\sum_{i=1}^{4} a_{i}^{2}+\sum_{i>j} 2 a_{i} a_{j} \exp \left(-\Gamma_{i}-\Gamma_{j}\right) \cos \left(\alpha_{i, f(i)}-\alpha_{j, f(j)}\right)
\end{aligned}
$$

When the number of photons put into input mode was $N_{\text {in }}$ counts/s, the number of the event "detection" $N_{\text {det }}$ is given as follows:

$$
N_{\text {det }}=N_{\text {in }} \eta \exp (-A) P_{1}+N_{\text {dark }} .
$$

The number of the events "nondetection" is given by $N_{\text {non }}$ $=N_{\text {total }}-N_{\text {det }}$. Under the assumption on the loss and the quantum efficiency given above, $N_{\text {total }}$ is given as follows:

$$
N_{\text {total }}=N_{\text {in }} \eta \exp (-A)+4 N_{\text {dark }} .
$$

As a result, $N_{n o n}$ is given as follows:

$$
N_{\text {non }}=N_{\text {in }} \exp (-A) \eta\left(1-P_{1}\right)+3 N_{\text {dark }} .
$$

Therefore, $P_{d e t}$ and $P_{n o n}$ which are the probabilities of the events detection and nondetection are written as follows:

$$
\begin{gathered}
P_{\text {det }}=\frac{N_{\text {det }}}{N_{\text {total }}}, \\
P_{\text {non }}=\frac{N_{\text {non }}}{N_{\text {total }}} .
\end{gathered}
$$

Using these relations, we can estimate the error rate of the quantum computation.

\section{B. The average error rate for the case "even"}

First, let us consider the average error rate for the six patterns of even inputs. For the even inputs, the result should be nondetection in an ideal case. Therefore, the error rate for the even inputs is given by the probability of the detection events for the even inputs. Using Eqs. (56), the averaged detection rate $N_{\text {det }}($ even $)$ can be described as follows:

$$
\begin{aligned}
N_{\text {det }}(\text { even })= & \frac{1}{6} \sum_{\{f(i)\}=\text { even }}\left(N _ { 0 } \left(\sum_{i=1}^{4} a_{i}^{2}+\sum_{i>j} 2 a_{i} a_{j}\right.\right. \\
& \times(-1)^{f(i)+f(j)} \exp \left(-\Gamma_{i}-\Gamma_{j}\right) \\
& \left.\left.\times \cos \left(\beta_{i}-\beta_{j}\right)\right\}+N_{\text {dark }}\right) \\
= & N_{0}\left\{\sum_{i=1}^{4} a_{i}^{2}-\sum_{i>j} \frac{2}{3} a_{i} a_{j} \exp \left(-\Gamma_{i}-\Gamma_{j}\right)\right. \\
& \left.\times \cos \left(\beta_{i}-\beta_{j}\right)\right\}+N_{\text {dark }},
\end{aligned}
$$

where $N_{0}=N_{i n} \eta \exp (-A)$, and we used the relation $\Sigma_{\{f(i)\}=\text { even }}(-1)^{f(i)+f(j)}=-2$ for $\forall i, j$. Neglecting the terms higher than the second order of error, E(even) $=P_{d e t}($ even $)$ is given as follows:

$$
\begin{gathered}
E(\text { even })=E_{1}(\text { even })+E_{2}(\text { even })+E_{3}(\text { even }) \\
E_{1}(\text { even })=\sum_{i=1}^{4} a_{i}^{2}-\sum_{i>j} \frac{2}{3} a_{i} a_{j} \cos \left(\beta_{i}-\beta_{j}\right), \\
E_{2}(\text { even })=\sum_{i>j} \frac{2}{3} a_{i} a_{j}\left[1-\exp \left(-\Gamma_{i}-\Gamma_{j}\right)\right] \cos \left(\beta_{i}-\beta_{j}\right),
\end{gathered}
$$




$$
E_{3}(\text { even })=\frac{N_{\text {dark }}}{N_{\text {total }}}
$$

where $E_{1}($ even $), E_{2}($ even $)$, and $E_{3}($ even $)$ describes the effect of systematic errors (systematic errors of beam splitting ratio, and imperfect initialize of the phase), the effect of phase relaxation, and the error caused by the dark count, respectively. The neglected terms are for the second order of these terms.

\section{The average error rate for the "uniform" case}

Next we consider the case where the result is a nondetection for uniform input. For the uniform inputs, the result should ideally be detection. Therefore, the error rate for the uniform inputs is given by the probability of the nondetection events for the uniform inputs. There are two uniform input patterns: one is filled with 0's and the other with 1's. Using Eq. (60), the average of E(uniform) $=P_{\text {non }}($ uniform $)$ for these two cases is calculated as follows:

$$
\begin{gathered}
\text { E }(\text { uniform })= \\
E_{1}(\text { uniform })+E_{2}(\text { uniform }) \\
+E_{3}(\text { uniform }), \\
E_{1}(\text { uniform })=1-\sum_{i=1}^{4} a_{i}^{2}-\sum_{i>j} a_{i} a_{j}\left[\cos \left(\beta_{i}-\beta_{j}\right)+\cos \left(\beta_{i}\right.\right. \\
\left.\left.+\gamma_{i}-\beta_{j}-\gamma_{j}\right)\right], \\
E_{2}(\text { uniform })=\sum_{i>j} a_{i} a_{j}\left\{\left[1-\exp \left(\Gamma_{i}-\Gamma_{j}\right)\right] \cos \left(\beta_{i}-\beta_{j}\right)\right. \\
\left.+\cos \left(\beta_{i}+\gamma_{i}-\beta_{j}-\gamma_{j}\right)\right\}, \\
E_{3}(\text { uniform })=\frac{3 N_{\text {dark }}}{N_{\text {total }}} .
\end{gathered}
$$

Hence $E_{1}$ (uniform), $E_{2}$ (uniform), and $E_{3}$ (uniform) show the effects of systematic errors, phase relaxation, and dark count, respectively.

\section{The estimation of $N_{\text {total }}$}

We estimated the total count $N_{\text {total }}$ as follows. Adding $3 N_{d e t}($ even $)$ to $N_{\text {det }}($ uniform $)$, we have

$$
\begin{aligned}
& 3 N_{\text {det }}(\text { even })+N_{\text {det }}(\text { uniform }) \\
& =3\left[N _ { 0 } \left(\sum_{i=1}^{4} a_{i}^{2}-\sum_{i>j} \frac{2}{3} a_{i} a_{j}\left[\exp \left(-\Gamma_{i}-\Gamma_{j}\right)\right]\right.\right. \\
& \left.\left.\quad \times \cos \left(\beta_{i}-\beta_{j}\right)\right)+N_{\text {dark }}\right]+N_{0}\left(\sum_{i=1}^{4} a_{i}^{2}+\sum_{i>j} 2 a_{i} a_{j}\right. \\
& \left.\quad \times\left[\exp \left(-\Gamma_{i}-\Gamma_{j}\right)\right] \cos \left(\beta_{i}-\beta_{j}\right)\right)+N_{\text {dark }}
\end{aligned}
$$

TABLE I. Estimated $a_{i j}$.

\begin{tabular}{llll}
\hline \hline$i$ & $a_{i}$ & \multicolumn{1}{c}{$\beta_{i}$} & \multicolumn{1}{c}{$\Gamma_{i}$} \\
\hline 1 & 0.274 & 0.600 & 0.0055 \\
2 & 0.250 & 0.244 & 0.0055 \\
3 & 0.257 & 0.157 & 0.015 \\
4 & 0.218 & 0.0 & 0.0055 \\
\hline \hline
\end{tabular}

$$
=N_{0}\left(4 \sum_{i=1}^{4} a_{i}^{2}\right)+4 N_{\text {dark }}
$$

where we neglected $\gamma_{j}$. As shown in Appendix B, $\sum_{i=1}^{4} a_{i}^{2}$ $\sim 1 / 4+\delta_{\theta}^{2} / 2$, where $\delta_{\theta}$ represents the systematic error of the beam splitters. When $\delta_{\theta}^{2}$ is sufficiently small, $\sum_{i=1}^{4} a_{i}^{2} \sim 1$ and Eq. (71) become $N_{0}+4 N_{\text {dark }}=N_{\text {total }}$. Therefore we used it in the quantitative analysis in the next section.

\section{E. Estimation of errors according to the sources in the four-bit $\mathrm{DJ}$ experiment}

We estimated $a_{j, f(j)}$ by the following procedure. If we set the shutter ST1 in Fig. 2 to open and ST2 to ST4 to closed, the intensity of the laser beam at the output port will be proportional to the following probability:

$$
P=\left|a_{i, f(i)}\right|^{2} \text {. }
$$

The difference between estimated $a_{i, 0}$ and $a_{i, 1}$ was less than $10^{-3}$, so we neglected the difference and put the average $a_{i}$ of them in Table I. We adapt the normalization $\sum_{i=1}^{4} a_{i}=1$ which is explained in Appendix B. ${ }^{2}$

We also estimated phase relaxation parameter $\Gamma_{j}$ as follows. In the experiment, we first measured the visibility for each of the Michelson interferometers. The visibility of the Michelson interferometer is given by

$$
\frac{2 a_{i} a_{j}}{a_{i}^{2}+a_{j}^{2}} \exp \left(-\Gamma_{i}-\Gamma_{j}\right)
$$

The estimated $-\Gamma_{i}-\Gamma_{j}$ for each interferometer is given in Table II. In the list, only $\Gamma_{i}+\Gamma_{j}$, which includes $\Gamma_{3}$, is twice as large as others. Therefore, we assumed that only $\Gamma_{3}$ is different from the others. As a result, we have $\Gamma_{1}=\Gamma_{2}$ $=\Gamma_{4} \sim 5.5 \times 10^{-3}, \Gamma_{3} \sim 1.5 \times 10^{-2}$ which were shown in Table I.

Next, we estimate the error $\beta_{i}$ according to the path difference. In the experiment, each interferometer was initialized to have a dark condition and we recorded the output count rates. Using Eqs. (53) and (55) with $\alpha_{i}$ and $\Gamma_{i}$, we estimated $\beta_{i}$ as shown in Table I.

\footnotetext{
${ }^{2}$ Strictly speaking, this normalization is not adaptable because we observed output mode 3 instead of output mode 1 . However, the error of the beam splitting ratio of BS1 was small in the experiment so that we did adapt this normalization.
} 
TABLE II. Estimation of $\Gamma_{j}$.

\begin{tabular}{llccc}
\hline \hline & \multicolumn{4}{c}{$2 a_{i} a_{j}$} \\
\cline { 3 - 5 } Interferometer & Visibility & $a_{i}^{2}+a_{j}^{2}$ & $\exp \left(-\Gamma_{i}-\Gamma_{j}\right)$ & $\Gamma_{i}+\Gamma_{j}$ \\
\hline Main (mode 2,4) & 0.97956 & 0.9904 & 0.9890 & $1.106 \times 10^{-2}$ \\
Sub1 (mode 1,2) & 0.9845 & 0.9956 & 0.9888 & $1.13 \times 10^{-2}$ \\
Sub2 (mode 3,4) & 0.9655 & 0.9859 & 0.9793 & $2.09 \times 10^{-2}$ \\
\hline \hline
\end{tabular}

The observed dark count rate in the experiment was 2.0 $\times 10^{3}$ counts $/ \mathrm{s}$, and $N_{\text {total }}$ was estimated to $6.00 \times 10^{5}$ counts/s in Sec. IV. Hence $N_{\text {dark }} / N_{\text {total }}$ was estimated to be 0.0033 .

Putting these parameters into Eqs. (63) to (65) and Eqs. (67) to (69), we can estimate the errors caused by each source. The estimated errors are listed in Table III. First, the error rate which appeared in the experiment was well reproduced by the sum of the estimated errors. For both of the answers, systematic error was the main cause of error: $72 \%$ in the case of even, and $70 \%$ in the case of uniform. In particular, the misadjustment of the path difference $\left(\delta_{\beta}\right)$ was the dominant systematic error. The error caused by the phase relaxation was three times larger for uniform than for even. This is consistent with the ratio of coefficients of $a_{i} a_{j}$ in Eqs. (64) and (68). The dark count has a similar relation as we expected from the Eqs. (65) and (69). The discrepancy between the sum of the estimated errors and the experimentally observed error rates may be mainly caused by the fact that we neglect the phase error between the state $f(i)=0$ and $f(i)=1$ by setting $\gamma_{i}=0$ in the analysis.

Using the estimated parameters in Table I, we simulated the photon detection probability using Eqs. (55) and (56). The simulated data are shown as empty squares in Fig. 5. These data points were symmetrical with respect to the center because we set $\gamma_{i}=0$. The main difference between the simulation and the experimental data may be caused by this simplification.

\section{ESTIMATION OF ERRORS IN THE EXPERIMENT OF THE DEUTSCH-JOZSA ALGORITHM WITH A $2^{k}$-BIT INPUT}

We can also construct an optical system for the DeutschJozsa algorithm with a $2^{k}$-bit input by preparing $2^{k}$ optical paths. In this section, we analyze the increase in error rate as a function of the input size $k$.

An optical system for $2^{k}$-bit input is shown in Fig. 6. A

TABLE III. The estimated errors according to the sources in the four-bit Deutsch Jozsa experiment.

\begin{tabular}{lcc}
\hline \hline Source of errors & Even & Uniform \\
\hline Systematic error & $1.82 \%$ & $5.01 \%$ \\
Phase relaxation & $0.37 \%$ & $1.10 \%$ \\
Dark count & $0.33 \%$ & $1.00 \%$ \\
Total & $2.52 \%$ & $7.11 \%$ \\
Experiment & $2.57 \%$ & $7.72 \%$ \\
\hline \hline
\end{tabular}

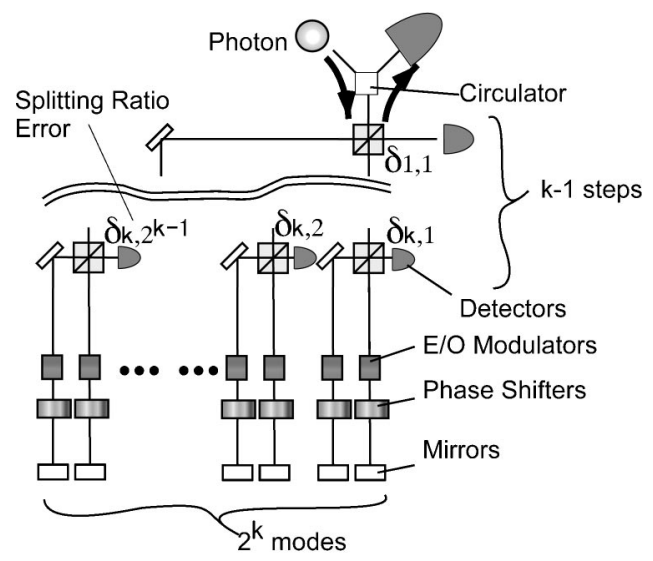

FIG. 6. A schematic diagram of the optical system for the Deutsch-Jozsa algorithm with $2^{k}$-bit input.

single photon wave function reaches a uniform superposition state in $2^{k}$ modes using $2^{k}-1$ beam splitters. In each mode $i$, the polarization is rotated only when $f(i)=1$, and the conditional $\pi / 2$ phase shift occurs at the wave plates. We use weak coherent light as the source of photons, therefore, we set the photon detectors at each of the output modes of beam splitters as shown in Fig. 6, and we consider the photon detection signal from the detector 1 as a detection event and those from the other detectors nondetection. This optical system is a straightforward expansion of the system shown in Fig. 4.

As described in Sec. V, we assume that the quantum efficiencies and dark count rates are the same for each other, and that the losses in each arm are balanced. The wave function at output mode 1 is the superpositon of the components given by Eqs. (52) and (53). Therefore, we can derive $N_{\text {det }}$ (the number of detection events per second.), $N_{\text {non }}$ (the number of nondetection events per second), and $N_{\text {total }}$ as follows:

$$
P=\sum_{i=1}^{2^{N}} a_{i}^{2}+\sum_{i>j} 2 a_{i} a_{j}\left[\exp \left(-\Gamma_{i}-\Gamma_{j}\right)\right] \cos \left(\alpha_{i, f(i)}-\alpha_{j, f(j)}\right),
$$

$$
\begin{gathered}
N_{\text {det }}=N_{0} P+N_{\text {dark }}, \\
N_{\text {non }}=N_{0}(1-P)+\left(2^{k}-1\right) N_{\text {dark }}, \\
N_{\text {total }}=N_{0}+2^{k} N_{\text {dark }} .
\end{gathered}
$$

For the even inputs, the result should be nondetection in an ideal case. Therefore, the error rate for the even inputs is given by the probability of the detection events for the even inputs. Using these equations, $E($ even $)=P_{\text {det }}($ even $)$, which is the probability of the detection event for the even input, is given as follows: 


$$
\begin{gathered}
E(\text { even })=\frac{N_{0}}{N_{\text {total }}}\left(\sum_{i=1} a_{i}^{2}-\frac{2}{2^{k}-1} \sum_{i>j} a_{i} a_{j}\right. \\
\left.\quad \times\left[\exp \left(-\Gamma_{i}-\Gamma_{j}\right)\right] \cos \left(\beta_{i}-\beta_{j}\right)\right)+\frac{N_{\text {dark }}}{N_{\text {total }}} \\
\begin{aligned}
& E_{1}(\text { even })+E_{2}(\text { even })+E_{3}(\text { even }) \\
& E_{1}(\text { even })= \sum_{i=1}^{2^{k}} a_{i}^{2}-\frac{2}{2^{k}-1} \sum_{i>j} a_{i} a_{j} \cos \left(\beta_{i}-\beta_{j}\right) \\
& E_{2}(\text { even })= \frac{2}{2^{k}-1} \sum_{i>j} a_{i} a_{j}\left[1-\exp \left(-\Gamma_{i}-\Gamma_{j}\right)\right] \\
& \times \cos \left(\beta_{i}-\beta_{j}\right) \\
& E_{3}(\text { even })=\frac{N_{\text {dark }}}{N_{\text {total }}}
\end{aligned}
\end{gathered}
$$

We neglected the terms higher than the second order of the sources. The derivation of the coefficient $2 /\left(2^{k}-1\right)$ is given in Appendix A. Here $E_{1}($ even $), E_{2}($ even $)$, and $E_{3}($ even $)$ correspond to the imperfect gates, the phase relaxation, and the darkcount, respectively.

First let us consider the effect of systematic errors, $E_{1}($ even $)$. We assume that a beam splitter $\mathrm{BS}(i, j)$ is representated by the transformation $R\left(\pi / 2+\delta_{i, j}\right)$ where $R$ is given in Eq. (2). In this case, as shown in Appendix B, $\sum_{i=1}^{2^{k}} a_{i}^{2}$ and $\sum_{i>j} a_{i} a_{j}$ can be approximated as follows.

$$
\begin{gathered}
\sum_{i=1}^{2^{k}} a_{i}^{2}=\frac{1+k \delta_{\theta}^{2}}{2^{k}} \\
\sum_{i>j} a_{i} a_{j}=\frac{2^{k}-1-k \delta_{\theta}^{2}}{2^{k+1}}
\end{gathered}
$$

where

$$
\delta_{\theta}^{2}=\frac{1}{k} \sum_{i=1}^{k} \frac{1}{2^{i-1}} \sum_{j=1}^{2^{i-1}} \sin ^{2} \delta_{i, j} .
$$

We also introduce a parameter $\delta_{\beta}$ as follows which represents the systematic error of the path difference.

$$
\cos \left(\beta_{i}-\beta_{j}\right) \simeq 1-\delta_{\beta}^{2}
$$

Using Eqs. (83) to (85), $E_{1}($ even) can be calculated as follows:

$$
E_{1}(\text { even }) \simeq \frac{k \delta_{\theta}^{2}}{2^{k-1}}+\frac{\delta_{\beta}^{2}}{2^{k}}
$$

Next, let us consider the error caused the phase relaxation, $E_{2}(e v e n)$. In the previous section, we listed the following
TABLE IV. The errors categorized according to the sources in the Deutsch-Jozsa quantum computer with $2^{k}$-bit input.

\begin{tabular}{lcc}
\hline \hline Source of errors & Even & Uniform \\
\hline Imperfect Gate & $\frac{k \delta_{\theta}^{2}}{2^{k-1}}+\frac{\delta_{\beta}^{2}}{2^{k}}$ & $\delta_{\beta}^{2}$ \\
Phase Relaxation & $\frac{1-\exp (-(4 k+8) \Gamma)}{2^{k}}$ & $1-\exp (-(4 k+8) \Gamma)$ \\
Dark count & $\frac{N_{\text {dark }}}{N_{\text {total }}}$ & $\frac{\left(2^{k}-1\right) N_{\text {dark }}}{N_{\text {total }}}$ \\
\hline \hline
\end{tabular}

sources: (a) the surface roughness of the optics, (b) the fluctuation of the reflactive index of the air, and (c) nonperfect overlap of the wave front because of the misalignment of the optics. The effect of source (b) can be eliminated by putting the whole system into a vaccum. The source (c) is a matter of the alignment of the optics and is independent of the input size. Here we analyze the effect of the increase in the number of optics in an optical path. As we saw in Sec. III, the phase relaxation parameter $\Gamma_{i}$ will increase linearly with the number of optics in a path. The number of optics in a path is $2 k+4$. When we assume a phase relaxation parameter caused by an optics is $\Gamma$, we can calculate $E_{2}$ (even) as follows:

$$
E_{2}(\text { even }) \sim \frac{1-\exp [-(4 k+8) \Gamma]}{2^{k}}
$$

The effect of dark count for the error rate $E_{3}$ (even)is constant for $k$. We summarized these results in Table IV

Next, let us calculate the error rate for the uniform inputs. For the uniform inputs, the result should be detection in an ideal case. Therefore, the error rate for the uniform inputs is given by the probability of the nondetection events for the uniform inputs. Therefore, E(uniform) is given by $P_{n o n}$ (uniform), which is an average of the probabilities for the nondetection events with uniform inputs. Hence,

$$
\begin{gathered}
\text { E (uniform })=P_{\text {non }}(\text { uniform }) \\
=\frac{N_{0}}{N_{\text {total }}}\left\{1-\sum_{i=1}^{2^{k}} a_{i}^{2}-\sum_{i>j} 2 a_{i} a_{j}\left[\exp \left(-\Gamma_{i}-\Gamma_{j}\right)\right]\right. \\
\left.\times \cos \left(\beta_{i}-\beta_{j}\right)\right\}+\frac{2^{k}-1}{N_{\text {total }}} N_{\text {dark }} \\
\sim E_{1}(\text { uniform })+E_{2}(\text { uniform })+E_{3}(\text { uniform }), \\
E_{1}(\text { uniform })=1-\sum_{i=1}^{2^{k}} a_{i}^{2}-\sum_{i>j} 2 a_{i} a_{j} \cos \left(\beta_{i}-\beta_{j}\right),
\end{gathered}
$$




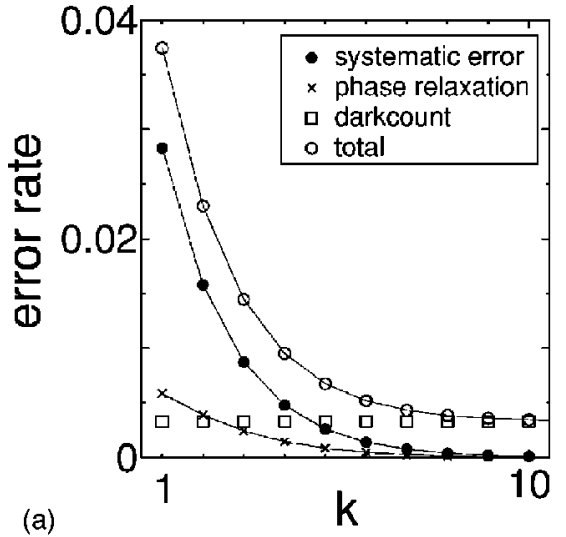

(a)

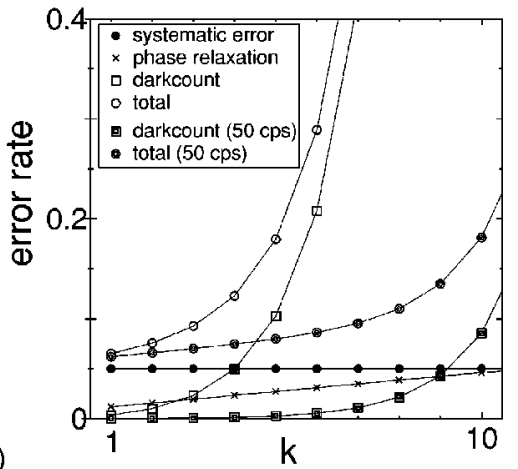

FIG. 7. The estimated error rate caused by the systematic errors, phase relaxation, and dark counts of the photon detectors in the optical system of the DJ algorithm with $2^{k}$-bit input.

$$
\begin{gathered}
E_{2}(\text { uniform })=\sum_{i>j} 2 a_{i} a_{j}\left[\left(-\exp \left(\Gamma_{i}-\Gamma_{j}\right)\right] \cos \left(\beta_{i}-\beta_{j}\right),\right. \\
E_{3}(\text { uniform })=\frac{2^{k}-1}{N_{\text {total }}} N_{\text {dark }}
\end{gathered}
$$

where $E_{1}$ (uniform), $E_{2}$ (uniform), and $E_{3}$ (uniform) correspond to the effect of imperfect gates, phase relaxation, and the dark count, respectively. Using Eqs. (83) and (84), $E_{1}$ (uniform) is given by

$$
E_{1}(\text { uniform }) \sim \delta_{\beta}^{2} .
$$

The effect of phase relaxation $E_{2}$ (uniform) can be written as follows:

$$
E_{2}(\text { uniform }) \sim 1-\exp [-(4 k+8) \Gamma] .
$$

The effect of dark count $E_{3}$ (uniform) is given by $\left(2^{k}\right.$ $-1) N_{\text {dark }} / N_{\text {total }}$. We also summarize these results in Table IV.

The estimated error rates for the even and uniform inputs are shown in Figs. 7(a) and 7(b). These graphs are plotted using the parameters found in the analysis of the four-bit experiment in Sec. V: $\delta_{\theta}^{2}=0.0033, \delta_{\beta}^{2}=0.05, \Gamma=0.00098$, and $N_{\text {dark }} / N_{\text {total }}=0.0033$. The number of input digits is given by $2^{k}$. In other words, the number of qubits which is simulated in the system is $k+1$.

In Fig. 7(a), the errors caused by imperfect gates (solid circles) and phase relaxation (crosses) decrease rapidly with the increase of $k$, and the error rate is almost given by the dark count (empty squares) when $k$ is large. At first glance, this decrease in the error rate may seem unreasonable, however it can be explained as follows. For the even inputs, the result should be nondetection in an ideal case. Therefore, the error rate for the even inputs is given by the probability of the detection events for the even inputs. Let us consider a situation where the phases of the wave functions are at random and no quantum interference occurs. When the size is $k=2$ and the system has four output ports, the detection probability at an output port is given by 0.25 , which is the error rate for an even input. When the size of the system increases, the number of the output mode increases to $2^{k}$ and the detection probability at an output mode is proportional to $2^{-k}$. This is why the error rate decreases with an increase of $k$.

Figure 7(b) shows the estimated error rate for uniform input, or the probability of a nondetection event with uniform inputs, plotted as empty circles. The error rate caused by dark count (empty squares) rapidly increases and is dominant with a large $k$. This is because the number of detectors which are set at all the output ports increases as $2^{k}$ with size $k$. The dark counts of the detectors except D1 increases the nondetection events.

It is also interesting that the error caused by phase relaxation (crosses) linearly increases in this region but not so rapidly, and the effect of systematic errors is constant. Therefore, the best way to improve the error rate is to decrease the dark count. We observed 2000 counts/s dark counts in the four-bit DJ experiment, however, we can decrease the rate down to 50 counts/s using commercially available detectors (for example, a special version of SPCM-AQ by EG \& G) and tight shielding for stray photons. We plotted the error rates with this small darkcount rate (50 counts/s) using double squares and the total error rates by double circles. The result shows that just by improving the dark count rates, we can perform the DJ algorithm experiment with a $2^{10}$ input, which is equivalent to 11 qubits, with less than a $20 \%$ total error rate.

It is also possible to decrease the error rate caused by the darkcount using a single photon source. One can create a pair of photons by use of spontaneous parametric down conversion (SPDC). By inputting one of them to the input port of the computer and with the other photon detected by a highquantum-efficiency single-photon counting system $[15,16]$, we can determine the time when the computation is performed. In this case, we do not need detectors except for detector D1 in Fig. 6 when the loss of the photon in the system is negligible. In reality, the correlation of the photon pair created by SPDC is not so high, however, we can improve the error rate by employing coincident counting between the detectors set at the output modes and the detector which determine when the computation is performed. 


\section{CONCLUSION}

In this paper, we have reported the quantitative analysis of the source of errors in linear optics computation. First we categorized the error in a simple one-qubit linear optics quantum computer according to the sources: systematic errors in quantum gates, phase relaxation, amplitude dumping, and misreadout. Then we estimated the effect of each error source in the four-bit DJ experiment, and succeeded in reproducing the error rate which appeared in the experiment by using the sum of the estimated error rates according to the sources. We also estimated the errors which occur in the optical system for the $2^{k}$-bit Deutsch-Jozsa algorithm. We found that the error is almost constant for the answer "not even," however, it increases exponentially with $k$ for the answer "not uniform." The main source of the error is caused by the dark counts of the photon detectors. We also found that the demonstration of quantum computation of 11 qubits using linear optics is achievable with the present technique used in the four-bit DJ experiment with commercially available detectors with smaller dark counts (50 counts/s).

With 11 qubits it is possible to realize several important proposals for quantum computation experimentally. For example, a experimental demonstration of DiVincenzo's error correcting code [17] is possible using six qubits. The factoring of small numbers using Shor's quantum algorithm [18] will be also possible within 11 qubits. It was confirmed in this paper that linear optics quantum computation is a practical test bed for these proposals.

Another interesting result of this paper is that the effect of the error sources strongly depended on the answers (in this case, "not even" and "not uniform') of the quantum computation. This result suggests the possibility of designing an algorithm whose results are not effected so much by the sources of error.

\section{ACKNOWLEDGMENTS}

The author would like to thank Dr. Toshiro Isu for his continuous discussion on this paper and Tetsuo Ogama for his support. The author is also grateful to the members of the Quantum Devices Department at Mitsubishi Electric, the members of the Kansai Society of Quantum Computation, and Professor Keiji Sasaki for their fruitful comments. I also wish to thank Professor Nobuyuki Imoto who encouraged me to study this issue.

\section{APPENDIX A: DERIVATION OF THE COEFFICIENT $2 /\left(2^{k}-1\right)$}

From Eq. (75),

$$
\begin{aligned}
N_{d e t}= & N_{0}\left(\sum_{i=1}^{2^{N}} a_{i}^{2}+\sum_{i>j} 2 a_{i} a_{j}\left[\exp \left(-\Gamma_{i}-\Gamma_{j}\right)\right]\right. \\
& \left.\times \cos \left(\alpha_{i, f(i)}-\alpha_{j, f(j)}\right)\right)+N_{\text {dark }} .
\end{aligned}
$$

Calculating the average of $N_{\text {det }}$ for the case $\{f(l)\}$ is even ( ${ }_{2} C_{2^{k-1}}$ combinations),

$$
\begin{aligned}
\sum_{\{f(l)\}} \frac{N_{d e t}}{{ }_{2^{k}} C_{2^{k-1}}}= & N_{0}\left[\sum_{i=1}^{2^{N}} a_{i}^{2}+\frac{1}{{ }_{2} C_{2^{k-1}}} \sum_{i>j} 2 a_{i} a_{j} \exp \left(-\Gamma_{i}\right.\right. \\
& \left.\left.-\Gamma_{j}\right)\left(\sum_{\{f(l)\}} \cos \left(\alpha_{i, f(i)}-\alpha_{j, f(j)}\right)\right)\right]+N_{\text {dark }} .
\end{aligned}
$$

When we assume $\beta_{i}-\beta_{j}=0$ and $\gamma_{j}=0$, We have the following equation:

$$
\begin{aligned}
F(i, j) & \equiv \sum_{\{f(l)\}} \cos \left(\alpha_{i, f(i)}-\alpha_{j, f(j)}\right) \\
& =\sum_{\{f(l)\}}(-1)^{f(i)+f(j)} .
\end{aligned}
$$

The meaning of this equation is summing $(-1)^{f(i)+f(j)}$ for all $\{f(l)\}$ which is even when an $i, j$ pair is given. There are ${ }_{2}^{k-2} C_{2^{k}-1}$ patterns of even $\{f(l)\}$ for the cases where $f(i)$ is not equal to $f(j)$, i.e., $\{f(i), f(j)\}=\{1,0\}$ or $\{0,1\}$. In such cases, $(-1)^{f(i)+f(j)}=-1$. On the other hand, there are ${ }_{2}{ }_{-2} C_{2^{k}}$ commutations for the case $\{f(i), f(j)\}=\{0,0\}$ or $\{1,1\}$. In such cases, $(-1)^{f(i)+f(j)}=+1$. Therefore, one can calculate Eq. (A3) as follows:

$$
F(i, j)=-2 \frac{\left(2^{k}-2\right) !}{\left(2^{k}-1\right) !\left(2^{k-1}\right) !} .
$$

Dividing by ${ }_{2}{ }^{k} C_{2^{k-1}}$ and multipling by 2 (the coefficient of $a_{i} a_{j}$ in the original equation), we finally have the coefficient:

$$
-\frac{2}{2^{k}-1}
$$

\section{APPENDIX B: THE DERIVATION OF $\sum_{i=1}^{2^{k}} a_{i}, \sum_{i=1}^{2^{k}} a_{i}^{2}$ AND $\sum_{i>j} a_{i} a_{j}$}

Here we derive $\sum_{i=1}^{2^{k}} a_{i}, \sum_{i=1}^{2^{k}} a_{i}^{2}$ and $\sum_{i>j} a_{i} a_{j}$. We assume that the matrix of the beam splitter $\operatorname{BS}(i, j)$ is given by Eq. (2) with the parameter $\theta=\pi / 2+\delta_{i, j}$, where $\delta_{i, j}$ denotes the systematic error. In this case, $a_{1}, a_{2}, \ldots, a_{2^{k}}$ is given as follows:

$$
\begin{gathered}
a_{1}=\frac{1-\sin \delta_{1,1}}{2} \frac{1-\sin \delta_{2,1}}{2} \cdots \frac{1-\sin \delta_{k, 1}}{2}, \\
a_{2}=\frac{1-\sin \delta_{1,1}}{2} \frac{1-\sin \delta_{2,1}}{2} \cdots \frac{1+\sin \delta_{k, 1}}{2}, \\
\cdots \cdots, \\
a_{2^{k}}=\frac{1+\sin \delta_{1,1}}{2} \frac{1+\sin \delta_{2,2}}{2} \cdots \frac{1+\sin \delta_{k, 2^{k-1}}}{2},
\end{gathered}
$$


where we used $\cos ^{2}\left(\pi / 4+\delta_{i, j} / 2\right)=\left(1-\sin \delta_{i, j}\right) / 2$. Therefore, we can have the following relation:

$$
\sum_{i=1}^{2^{k}} a_{i}=1
$$

Next we calculate $\sum_{i=1}^{2^{k}} a_{i}^{2}$. Using Eqs. (B1) and (B2), we have

$$
\begin{aligned}
a_{1}^{2}+a_{2}^{2}= & \left(\frac{1-\sin \delta_{1,1}}{2}\right)^{2}\left(\frac{1-\sin \delta_{2,1}}{2}\right)^{2} \ldots\left(\frac{1-\sin \delta_{k-1,1}}{2}\right)^{2} \\
& \times \frac{1+\sin ^{2} \delta_{k, 1}}{2} .
\end{aligned}
$$

When we consider the terms up to the second order of $\delta_{i, j}$, we can approximate this equation as follows:

$$
a_{1}^{2}+a_{2}^{2} \sim \frac{1}{2}\left(\frac{1-\sin \delta_{1,1}}{2}\right)^{2}\left(\frac{1-\sin \delta_{2,1}}{2}\right)^{2} \ldots\left(\frac{1-\sin \delta_{k-1,1}}{2}\right)^{2}+\frac{\sin ^{2} \delta_{k, 1}}{2^{2 k-1}} .
$$

Similarly, we have $a_{3}^{2}+a_{4}^{2}$ as follows:

$$
a_{3}^{2}+a_{4}^{2} \sim \frac{1}{2}\left(\frac{1-\sin \delta_{1,1}}{2}\right)^{2}\left(\frac{1-\sin \delta_{2,1}}{2}\right)^{2} \ldots\left(\frac{1-\sin \delta_{k-1,1}}{2}\right)^{2}+\frac{\sin ^{2} \delta_{k, 2}}{2^{2 k-1}} .
$$

Thus, we have $\sum_{i=1}^{4} a_{i}^{2}$ as follows:

$$
\begin{aligned}
\sum_{i=1}^{4} a_{i}^{2} \sim & \frac{1}{4}\left(\frac{1-\sin \delta_{1,1}}{2}\right)^{2}\left(\frac{1-\sin \delta_{2,1}}{2}\right)^{2} \cdots\left(\frac{1-\sin \delta_{k-2,1}}{2}\right)^{2} \\
& +\frac{\sin ^{2} \delta_{k-1,1}}{2^{2 k-2}}+\frac{\sin ^{2} \delta_{k, 1}+\sin ^{2} \delta_{k, 2}}{2^{2 k-1}} .
\end{aligned}
$$

As a result, we have following equation:

$$
\sum_{i=1}^{2^{k}} a_{i}^{2} \sim \frac{1}{2^{k}}+\sum_{i=1}^{k} \sum_{j=1}^{2^{i-1}} \frac{\sin ^{2} \delta_{i, j}}{2^{k+i-1}} .
$$

Using the average of the systematic error for the beam splitters at $i$ steps $\delta_{i}^{2}=\left(\sum_{j=1}^{2^{i-1}} \sin ^{2} \delta_{i, j}\right) / 2^{i-1}$,

$$
\sum_{i=1}^{2^{k}} a_{i}^{2} \sim \frac{1}{2^{k}}+\frac{1}{2^{k}} \sum_{i=1}^{k} \delta_{i}^{2} .
$$

This equation suggests that an accidental systematic error of a beam splitter near the root of the tree of the paths causes a serious increase in error. Using parameter $\delta_{\theta}^{2}$ which is an average of $\delta_{i}^{2}$, we have following equation:

$$
\sum_{i=1}^{2^{k}} a_{i}^{2} \sim \frac{1}{2^{k}}+\frac{k}{2^{k}} \delta_{\theta}^{2}
$$

Using these results, $\sum_{i>j} a_{i} a_{j}$ is given as follows.

$$
\begin{aligned}
\sum_{i>j} a_{i} a_{j} & =\frac{1}{2}\left(\left(\sum_{i=1}^{2^{k}} a_{i}\right)^{2}-\sum_{i=1}^{2^{k}} a_{i}^{2}\right) \\
& =\frac{2^{k}-1-k \delta_{\theta}^{2}}{2^{k+1}}
\end{aligned}
$$

[1] D. Deutsch, Proc. R. Soc. London, Ser. A 400, 97 (1985).

[2] A. Ekert and R. Jozsa, Rev. Mod. Phys. 68, 733 (1996).

[3] D.P. Divincenzo, Science 270, 255 (1995).

[4] A. Barenco, D. Deutsch, A. Ekert, and R. Jozsa, Phys. Rev. Lett. 74, 4083 (1995).

[5] J.I. Cirac and P. Zoller, Phys. Rev. Lett. 74, 4091 (1995).

[6] B.E. Kane, Nature (London) 393, 133 (1998).

[7] N.A. Gershenfeld and I. Chuang, Science 275, 350 (1997).

[8] D.G. Cory, M.D. Price, and T.F. Havel, Physica D 120, 82 (1998).

[9] X. Zhou, D.W. Leung, and I.L. Chuang, e-print quant-ph/9906112.
[10] M. Reck, A. Zeilinger, H.J. Bernstein, and P. Berani, Phys. Rev. Lett. 73, 58 (1994).

[11] S. Takeuchi, in Proceedings of the Fourth Workshop on Physics and Computation: PhysComp96, edited by T. Toffoli (New England Complex Systems Institute, Boston, 1996), pp. 299302.

[12] N.J. Cerf, C. Adami, and P.G. Kwiat, Phys. Rev. A 57, R1477 (1998).

[13] D. Deutsh and R. Jozsa, Proc. R. Soc. London, Ser. A 439, 553 (1992).

[14] S. Takeuchi (unpublished).

[15] S. Takeuchi, J. Kim, Y. Yamamoto, and H.H. Hogue, Appl. Phys. Lett. 74, 1063 (1999). 
[16] J. Kim, S. Takeuchi, Y. Yamamoto, and H.H. Hogue, Appl. Phys. Lett. 74, 902 (1999).

[17] D.P. DiVincenzo and P.W. Shor, Phys. Rev. Lett. 77, 3260 (1998).
[18] P. W. Shor, in Proceedings of the 35th Annual Symposium on the Foundations of Computer Science, edited by S. Goldwasser (IEEE Computer Society, Los Alamitos, CA, 1994), p. 124. 